\title{
Evolving myelodysplastic syndrome in an HIV patient with history of anal cancer and chemotherapy
}

\author{
Mingyue Shi ${ }^{1} \cdot$ Xiaobang Hu${ }^{1} \cdot$ Mingyi Chen ${ }^{1}$ \\ Received: 13 July 2019 / Accepted: 11 September 2019 / Published online: 30 October 2019 \\ (C) Springer-Verlag GmbH Germany, part of Springer Nature 2019
}

\begin{abstract}
The incidence of therapy-related myelodysplastic syndromes (t-MDS) has been increasing with the widespread use of highly active antiretroviral therapy (HAART) therapy for HIV and chemotherapy for AIDS-related cancers. The classical dysplastic features in the granulocytes and megakaryocytes may not be easily appreciated. The most reliable distinguishing feature between the hematopoietic dysplasia of t-MDS and that of HIV infection rests on the identification of MDS-related cytogenetic aberrations. Here we report a patient with well-controlled HIV and history of chemotherapy for invasive anal squamous cell carcinoma who developed high-risk t-MDS with complex chromosome abnormalities. Our study emphasizes the importance of diagnosis of MDS in HIV-infected patients, even in the absence of dysplasia, if there are typical cytogenetics changes of MDS. Therefore, the early diagnosis and intervention of t-MDS in HIV-positive patients are critical in the treatment of this aggressiveness disease.
\end{abstract}

Keywords Therapy-related myelodysplastic syndromes (t-MDS) - Acquired immune deficiency syndrome · HIV · Cancer . Chemotherapy

A 60-year-old white male presented with worsening fatigue for 2 weeks. The patient had a longstanding history of HIV infection and clinical complications of acquired immune deficiency syndrome (AIDS) including cryptococcal meningitis. He was diagnosed with invasive anal squamous cell carcinoma 6 years ago and received mitomycin and radiation therapy. His HIV infection was well controlled by highly active antiretroviral therapy (HAART) treatment. The most recent CD4 T cell count was $299 / \mu$ L. Peripheral blood smear showed pancytopenia. Complete blood count $(\mathrm{CBC})$ : WBC $2.91 \times 10^{9} / \mathrm{L}$, neutrophils $34 \%$, lymphocytes $61 \%$, monocytes $4 \%$, eosinophils $1 \%$, basophils $0 \%$, RBC $2.81 \times 10^{12} / \mathrm{L}, \mathrm{HGB}$ $7.2 \mathrm{~g} / \mathrm{dL}, \mathrm{HCT} 22.5 \%$, MCV $80.1 \mathrm{Fl}$, and PLT $14 \times 10^{9} / \mathrm{L}$. Bone marrow biopsy revealed hypercellular $(80 \%$

Mingyi Chen

mingyi.chen@utsouthwestern.edu

Mingyue Shi

Mingyue.Shi@UTSouthwestern.edu

Xiaobang $\mathrm{Hu}$

XIAOBANG.HU@phhs.org

1 Department of Pathology, UT Southwestern Medical Center BioCenter, EB3.234A; 2330 Inwood Rd., Dallas, TX 75390, USA cellularity) marrow with left-shifted granulopoiesis, clusters of blasts, megakaryocytic and erythroid dysplasia (Fig. 1a: blue arrow large cells are blasts, red arrow indicates irregular nuclear dysplastic erythroid precursor; Fig. 1b: green arrow indicates dysplastic megakaryocyte, blue arrow indicates immature mononuclear cells/blasts), and reactive lymphoid aggregates. The immunostaining of CD34 highlighted interstitially increased clusters of blasts (34\%) (Fig. 1c). Flow cytometry report revealed a 58\% maturing granulocytic elements with decreased orthogonal light scatter properties (suggesting hypogranularity) and with an unusual maturation spectrum, and an increased distinct myeloblasts population with immunophenotypical aberrancy (CD34+/CD117+/ CD33+/subset CD56+/CD7dim+/HLA-DR+) (Fig. 1d, highlight in red). Cytogenetic analysis found the following complex karyotype of multiples chromosome abnormalities. 43,X,-Y,5, del(7)(q22q36), inv(12)(p13q15), der(13)del(13)(q14q34)$\mathrm{t}(5 ; 13)(\mathrm{p} 13 ; \mathrm{q} 14),-17[5] / 43$, idem, - inv(12), + $\operatorname{der}(12) \mathrm{t}(12 ; 17)(\mathrm{p} 12 ; \mathrm{q} 21)[5] / 43$, idem,-inv(12),+ $\operatorname{der}(12) \operatorname{inv}(12)(p 13 q 24.1) \operatorname{add}(12)(p 13)[5]$ (Fig. 1e).

The patient was diagnosed with therapy-related myelodysplasia (t-MDS) and treatment with azacitidine was initiated. The patient developed progressive pancytopenia and passed away in 3 months. Dysplastic changes to the marrow are a common finding in advanced 


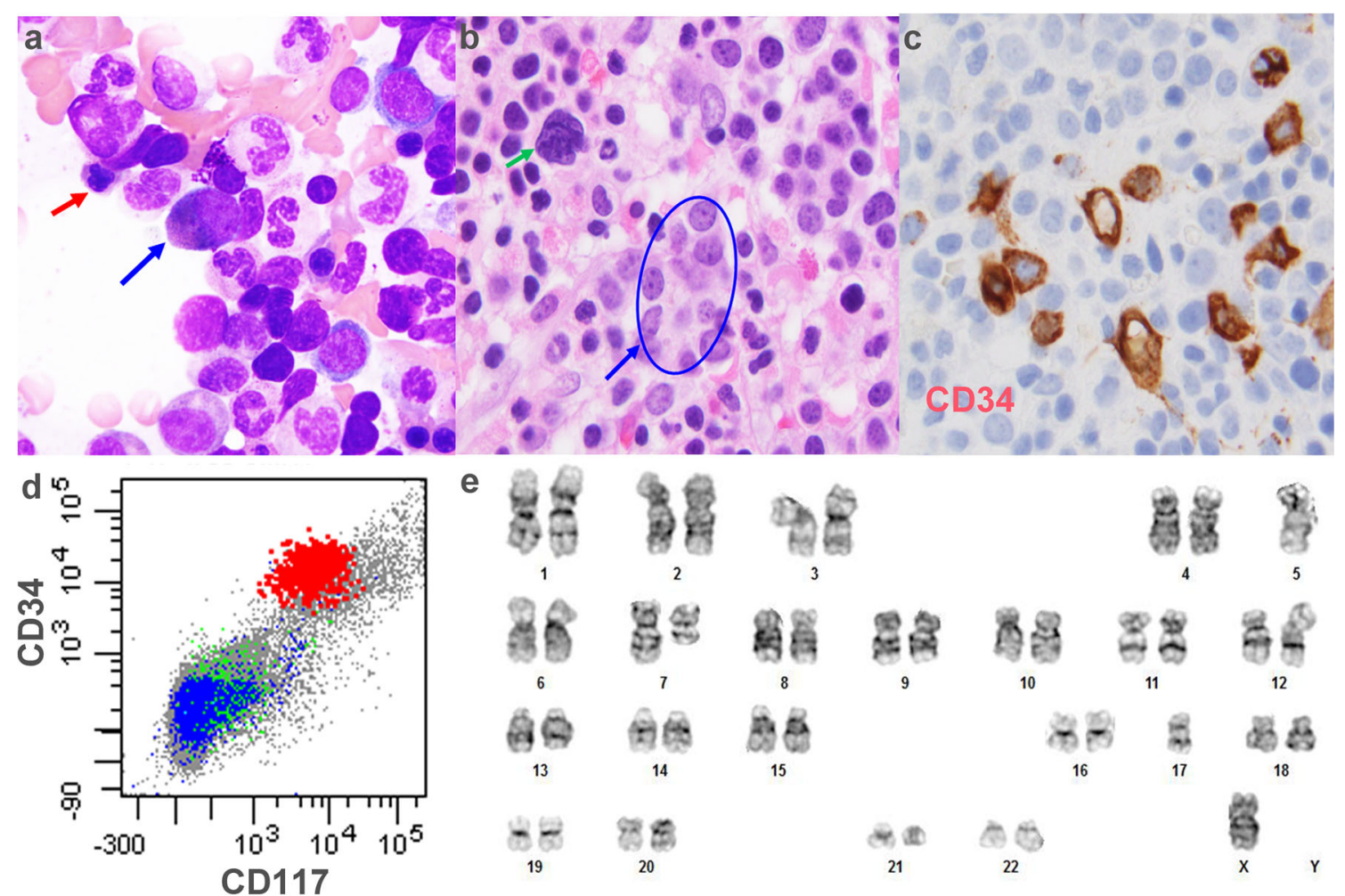

Fig. 1 a Morphological, immunophenotypical and cytogentic pathological findings of the case, b Bone marrow biopsy, $\mathbf{c}$ immunostaining of CD34, $\mathbf{d}$ flow cytometry report, and e cytogenetic analysis of a 60 -year-old HIV patient

HIV infection on HAART therapy [1]. This case represents a patient with well-controlled HIV and history of chemotherapy who developed high-risk t-MDS with complex chromosome abnormalities. The incidence of t-MDS has been increasing with the widespread use of HAART for HIV and chemotherapy for AIDS-related cancers $[2,3]$. The most reliable distinguishing feature between the hematopoietic dysplasia of t-MDS and that of HIV infection rests on the identification of MDSrelated cytogenetic aberrations $[4,5]$. The early diagnosis and intervention of t-MDS in HIV-positive patients are critical in treatment of this aggressiveness disease.

\section{Compliance with ethical standards}

Conflict of interest The authors declare that they have no conflict of interest.

\section{References}

1. Williamson BT, Leitch HA (2016) Higher risk myelodysplastic syndromes in patients with well-controlled HIV infection: clinical features, treatment, and outcome. Case Rep Hematol 2016:8502641

2. Fang RC, and Aboulafia DM (2016) HIV infection and myelodysplastic syndrome/acute myeloid leukemia. https://doi.org/ 10.1007/978-3-319-26857-6_10

3. Kaner J, Thibaud S, Sridharan A, Assal A, Polineni R et al (2016) $\mathrm{HIV}$ is associated with a high rate of unexplained multilineage cytopenias and portends a poor prognosis in myelodysplastic syndrome (MDS) and acute myeloid leukemia (AML). Blood 2016(128):4345

4. Takahashi K, Yabe M, Shapira I, Pierce S, Garcia-Manero G, Varma M (2012) Clinical and cytogenetic characteristics of myelodysplastic syndrome in patients with HIV infection. Leuk Res 36:1376-1379

5. Rieg S, Lubbert M, Kern WV, Timme S, Gartner F et al (2009) Myelodysplastic syndrome with complex karyotype associated with long-term highly active antiretroviral therapy. Br J Haematol 145: $670-673$

Publisher's note Springer Nature remains neutral with regard to jurisdictional claims in published maps and institutional affiliations. 